\title{
Distributed Interference Alignment and Power Control for Wireless MIMO Interference Networks
}

\author{
Hamed Farhadi, Chao Wang, and Mikael Skoglund \\ Communication Theory Laboratory, School of Electrical Engineering \\ Royal Institute of Technology (KTH), Stockholm, Sweden \\ Emails: \{farhadih, chaowang, skoglund $\}$ kth.se
}

\begin{abstract}
This paper considers joint transceiver design and power control for $K$-user multiple-input multiple-output (MIMO) interference networks. Each source intends to communicate with its corresponding destination at a fixed data rate. Only local channel side information (i.e. knowledge related to the channels directly connected to a terminal) is available at each terminal. We propose iterative algorithms to perform power control to guarantee successful communication while designing transmitter beamforming matrices and receiver filtering matrices according to the interference alignment concept. The proposed algorithms can exhibit a substantial performance improvement compared to the conventional orthogonal transmission schemes.
\end{abstract}

\section{INTRODUCTION}

Designing efficient transmission schemes for wireless interference networks, which refer to communication networks in which multiple source-destination pairs share the radio spectrum, is a challenging problem. Due to the broadcast nature of wireless transmission medium, the reception at each destination can be potentially interfered by signals sent from unintended sources. Conventional interference management strategies (e.g. time-division-multiple-access, TDMA, or frequency-division-multiple-access, FDMA) tend to orthogonalize the transmissions of different source-destination pairs. This requirement leads to the fact that at each destination, the subspaces of different interference signals are orthogonal to that of the desired signal and also orthogonal to each other. Thus, interference is avoided at the cost of low spectral efficiency. Hence, it was believed that the performance of wireless networks is limited by interference in general.

However, the elegant interference alignment concept [1], [2] reveals that with proper transmission design, different interference signals at each destination can in fact be aligned together, such that more radio resources can be assigned to the desired transmission. For instance, consider a multiple-input multiple-output (MIMO) interference network with more than two source-destination pairs. In certain cases (e.g. a threeuser network), the sources can perform linear beamforming to send their signals simultaneously in such a way that at each destination interference signals are aligned together to span only half of the available signal space (in the spatial domain). Thus, the interference can be completely eliminated with simple linear zero-forcing filters [2]. At the high-SNR region, each source-destination pair can potentially attain half of its interference-free achievable transmission rate.

Although interference alignment can substantially achieve better performance compared to the orthogonal transmission strategies, its realization can be rather difficult. For instance, normally the global channel state information (CSI) is required to be perfectly known at all terminals. Clearly, acquiring such channel knowledge is a challenging problem in practice. In most cases, it may be more convenient for each terminal to obtain the CSI of the channels directly connected to it (i.e. local CSI). To handle this issue, an iterative solution for distributed interference alignment is proposed in [3]. However, the total interference may not be completely eliminated at each destination. Thus, the communication performance of each source-destination pair (in general determined by signalto-interference-plus-noise ratio, SINR, at destination) actually depends on the transmission powers of all sources. This leads to a competitive situation: when each source tries to increase its transmission power to compensate interference at its intended destination, it in fact also increases the interference to unintended destinations. Ignoring this dependency in signalling design may cause unnecessarily extra transmission powers or unsatisfactory communication quality.

In certain applications such as voice and video communications, fixed-rate data transmission is desired [4]. Therefore, proper power control (see e.g. [5]-[10]) is required to efficiently utilize available resources to fulfill the demanded communication quality. Consider a MIMO interference network, directly applying the power control schemes while multiple source-destination pairs are non-orthogonally activated may either be infeasible or lead to large power requirements. Thus, to guarantee successful transmission at a fixed rate between each source-destination pair, conventionally, power control is carried out when the transmissions of different sources are orthogonallized. This may not be a spectrally efficient transmission strategy. In this paper, however, we design power control while the interference alignment technique is performed. More specifically, we propose two iterative algorithms which try to both design transmitter beamforming/receiver filtering matrices to align and eliminate interference, and also allocate the minimum powers to realize desired fix-rate communications. Numerical evaluations show that these algorithms require substantially smaller powers, when compared to the conventional orthogonal transmission strategies.

The structure of this paper is as follows. Section II describes system model. In Section III, we elaborate on the iterative algorithms to conduct joint transceiver design and power control. Section IV provides numerical performance evaluations. Finally, Section V concludes this paper.

This is an author produced version of a paper published at the IEEE Wireless Communications and Networking Conference (WCNC'13),07 - 10 April 2013, Shanghai, China. This paper has been peer-reviewed but does not include the final publisher proof-corrections or proceedings pagination. 


\section{Multi-User Mimo Interference Network}

We consider a time-invariant MIMO interference network consisting of $K$ source-destination pairs. The sources and destinations are denoted as $\mathrm{S}_{k}$ and $\mathrm{D}_{k}(k \in\{1,2, \ldots, K\})$, and are equipped with $n_{k}^{\mathrm{S}}$ and $n_{k}^{\mathrm{D}}$ antennas, respectively.

\section{A. Linear Beamforming at Sources}

$\mathrm{S}_{k}$ sends $d_{k}$ independently encoded Gaussian codewords. The power of the $d$ th codeword $\left(d \in\left\{1, \ldots, d_{k}\right\}\right)$ is $p_{k}^{d}$. Let $\mathbf{V}_{k}$ be an $n_{k}^{\mathrm{S}} \times d_{k}$ transmitter beamforming matrix with column vectors $\mathbf{v}_{k}^{d}\left(d \in\left\{1, \ldots, d_{k}\right\}\right)$. The column vectors are the orthogonal basis of the transmitted signal space of $\mathrm{S}_{k}$. The transmitted signal of $S_{k}$ can be written as:

$$
\mathbf{x}_{k}=\mathbf{V}_{k} \overline{\mathbf{x}}_{k}
$$

where $\overline{\mathbf{x}}_{k}$ is a $d_{k} \times 1$ vector of transmitted codewords.

\section{B. Linear Filtering at Destinations}

Let $\mathbf{U}_{k}$ denote an $n_{k}^{\mathrm{D}} \times d_{k}$ receiver filtering matrix with column vectors $\mathbf{u}_{k}^{d}\left(d \in\left\{1, \ldots, d_{k}\right\}\right)$. The column vectors are the orthogonal basis of the desired signal subspace at $\mathrm{D}_{k}$. The filter output of $\mathrm{D}_{k}$ is as follows:

$$
\overline{\mathbf{y}}_{k}=\mathbf{U}_{k}^{*} \mathbf{y}_{k},
$$

where $\mathbf{A}^{*}$ denotes the conjugate transpose of matrix $\mathbf{A}$. When the sources are non-orthogonally activated, the filter output of $\mathrm{D}_{k}$ generally contains interference, i.e.

$$
\overline{\mathbf{y}}_{k}=\mathbf{U}_{k}^{*} \mathbf{H}_{k k} \mathbf{V}_{k} \overline{\mathbf{x}}_{k}+\sum_{l=1, l \neq k}^{K} \mathbf{U}_{k}^{*} \mathbf{H}_{k l} \mathbf{V}_{l} \overline{\mathbf{x}}_{l}+\mathbf{U}_{k}^{*} \mathbf{z}_{k},
$$

where $\mathbf{H}_{k l}$ is the channel matrix from $\mathrm{S}_{l}$ to $\mathrm{D}_{k}$, and $\mathbf{z}_{k}$ is the complex Gaussian noise $\mathbf{z}_{k} \sim \mathcal{C N}\left(\mathbf{0}, N_{0} \mathbf{I}_{n_{k}^{\mathrm{D}}}\right)$ in which $N_{0}$ is noise power at each destination, and $\mathbf{I}_{n_{k}^{\mathrm{D}}}$ is an $n_{k}^{\mathrm{D}} \times$ $n_{k}^{\mathrm{D}}$ identity matrix. In the considered network, it is desired that each source $\mathrm{S}_{k}$ can always communicate with its intended destination $\mathrm{D}_{k}$ with rate $R_{k}$. Specifically, through properly designed $\mathbf{V}_{k}, \mathbf{U}_{k}$, and $p_{k}^{d}$, successful communication between every source-destination pair should be guaranteed.

\section{Interference Alignment And Power Control}

Multiple antennas can be exploited to perform interference alignment in the spatial domain. The transmitted signals are beamformed by the sources in such a way that at each destination interference signals are aligned in the same subspace, which is distinct from the desired signal subspace. Consequently, the desired signals can be recovered by eliminating the interference with proper filtering. For general MIMO interference networks, interference alignment may not be always feasible. Some studies on the feasibility conditions can be found in [3], [11]. In the considered network, if $d_{k}$ $(\forall k \in\{1, \ldots, K\})$, are carefully chosen such that interference alignment is feasible, there exist transmitter beamforming and receiver filtering matrices that satisfy the following conditions:

$$
\begin{aligned}
\mathbf{U}_{k}^{*} \mathbf{H}_{k j} \mathbf{V}_{j} & =\mathbf{0}, \forall j \neq k: j, k \in\{1, \ldots, K\}, \\
\operatorname{rank}\left(\mathbf{U}_{k}^{*} \mathbf{H}_{k k} \mathbf{V}_{k}\right) & =d_{k}, \forall k \in\{1, \ldots, K\} .
\end{aligned}
$$

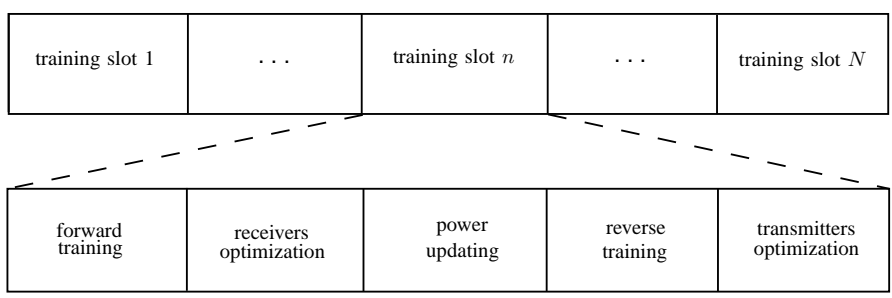

Fig. 1: CSI acquisition, transceiver design and power control

In general, perfect global CSI is required at all terminals to find a solution of this problem. Nevertheless, acquiring global CSI is difficult, and thus it is not considered in this paper. An iterative optimization of the transmitter beamforming and the receiver filtering matrices is proposed in [3] which demands only local CSI at each terminal. Applying this method incurs some interference to be remained in the desired signal subspace at each destination. Therefore, carelessly increasing each source's power may degrade the performance of the other source-destination pairs. On the other hand, this scheme mainly aims at maximizing the sum throughput of interference networks with fixed power budgets (i.e. a rate adaptation problem), rather than proving successful fixed-rate communication with minimized power requirements. Hence, an additional power control strategy is demanded in the considered network.

In this paper we permit each destination to estimate its local CSI from the training sequences sent by the sources. Also, to obtain local CSI at each source, the destinations transmit training sequences over reverse channels (channels from destinations to sources) which are separated from forward channels (channels from sources to destinations) in time via timedivision duplexing (TDD). Let $\overleftarrow{\mathbf{H}}_{k l}$ denote the channel matrix from $\mathrm{D}_{l}$ to $\mathrm{S}_{k}$. The reciprocity of the forward and the reverse channels is assumed, i.e. $\overleftarrow{\mathbf{H}}_{k l}=\mathbf{H}_{l k}^{*}(\forall l, k \in\{1,2, \ldots, K\})$. In the following, we briefly present the process of iterative CSI acquisition, transceiver design, and power control.

\section{A. CSI Acquisition, Transceiver Design, and Power Control}

The training and optimization scheme is an iterative procedure in which each iteration occurs during a training slot. There are $N$ training slots, and within each slot different tasks are performed as depicted in Fig. $1 . N$ is a design parameter and the accuracy of system design can be improved by setting $N$ with a larger value. Before the first training slot starts, $\mathrm{S}_{k}$ $(k \in\{1,2, \ldots, K\})$ randomly chooses its initial powers $p_{k}^{l}(0)$ $\left(l \in\left\{1, \ldots, d_{k}\right\}\right)$ and beamforming matrix $\mathbf{V}_{k}(0)$ such that $\left(\mathbf{V}_{k}(0)\right)^{*} \mathbf{V}_{k}(0)=\mathbf{I}_{d_{k}}$. The $n$th $(n \in\{1,2, \ldots, N\})$ training slot is composed of the following phases:

1) Forward training phase: The sources broadcast training sequences, and $\mathrm{D}_{k}$ estimates the following matrix:

$$
\mathbf{Q}_{k}(n-1)=\sum_{\substack{j=1 \\ j \neq k}}^{K} \sum_{\substack{d \\ j}}^{d_{j}} p_{j}^{d}(n-1) \mathbf{H}_{k j} \mathbf{v}_{j}^{d}(n-1)\left(\mathbf{v}_{j}^{d}(n-1)\right)^{*} \mathbf{H}_{k j}^{*} .
$$

This matrix is termed interference covariance matrix. 
2) Receiver optimization phase: Using $\mathbf{U}_{k}(n)$ to denote the receiver filtering matrix, the total power of remained interference at $\mathrm{D}_{k}$ is

$$
I F_{k}(n)=\operatorname{Tr}\left[\left(\mathbf{U}_{k}(n)\right)^{*} \mathbf{Q}_{k}(n-1) \mathbf{U}_{k}(n)\right],
$$

where $\operatorname{Tr}[\mathbf{A}]$ denotes the trace of matrix $\mathbf{A}$. To minimize $I F_{k}(n), \mathbf{u}_{k}^{d}(n)\left(\forall d \in\left\{1, \ldots, d_{k}\right\}\right)$ is chosen as follows [3]:

$$
\mathbf{u}_{k}^{d}(n)=\nu^{d}\left[\mathbf{Q}_{k}(n-1)\right],
$$

where $\nu^{d}[\mathbf{A}]$ is the eigenvector corresponding to the $d$ th smallest eigenvalue of matrix $\mathbf{A}$.

3) Power updating phase: In this phase, powers update according to the power control schemes which will be described in Section III-B. First, we will consider an iterative scheme which in each iteration, destination $\mathrm{D}_{k}$ computes the required transmission power of source $\mathrm{S}_{k}$, and informs $\mathrm{S}_{k}$ about how to update the transmission power via a feedback signal. We will provide the convergence proof of this scheme. Next, we will propose a simplified scheme which updates the powers once within each training slot. The convergence of the powers after consequent training slots is verified by numerical evaluations. For the implementation of this scheme, we will consider two feedback schemes. In the first scheme, each destination sends back the exact value to which the source transmission power should be updated. In the second one, each destination uses only a one-bit feedback signal to inform the corresponding source to either increase or decrease its power by a given value.

4) Reverse training phase: $D_{k}$ beamforms its training sequences, with a fixed power $p_{F}$ uniformly allocated to different sequences, using an $n_{k}^{\mathrm{D}} \times d_{k}$ matrix $\overleftarrow{\mathrm{V}}_{k}$. At the same time, $\mathrm{S}_{k}$ applies an $n_{k}^{\mathrm{S}} \times d_{k}$ filtering matrix $\overleftarrow{\mathbf{U}}_{k}$ to its received signal. If $\overleftarrow{\mathbf{V}}_{k}$ and $\overleftarrow{\mathbf{U}}_{k}$ are chosen to satisfy the following conditions

$$
\begin{aligned}
\overleftarrow{\mathbf{U}}_{k}^{*} \overleftarrow{\mathbf{H}}_{k j} \overleftarrow{\mathbf{V}}_{j} & =\mathbf{0}, \forall j \neq k: j, k \in\{1, \ldots, K\} \\
\operatorname{rank}\left(\overleftarrow{\mathbf{U}}_{k}^{*} \overleftarrow{\mathbf{H}}_{k k} \overleftarrow{\mathbf{V}}_{k}\right) & =d_{k}, \quad \forall k \in\{1, \ldots, K\}
\end{aligned}
$$

by setting $\mathbf{V}_{k}=\overleftarrow{\mathbf{U}}_{k}$ and $\mathbf{U}_{k}=\overleftarrow{\mathbf{V}}_{k}$, the conditions in (4) are also met. Again, due to the insufficient CSI, it is hard to directly find matrices to satisfy equations in (8). However, we can still take the advantage of the channel reciprocity. Specifically, $\mathrm{D}_{k}$ set its beamforming matrix as $\overleftarrow{\mathbf{V}}_{k}(n)=\mathbf{U}_{k}(n)$, in which $\mathbf{U}_{k}(n)$ is obtained by (7). Thus, the reverse interference covariance matrix measured at $\mathrm{S}_{l}$ $(l \in\{1,2, \ldots, K\})$ is

$$
\overleftarrow{\mathbf{Q}}_{l}(n-1)=\sum_{j=1, j \neq l}^{K} \frac{p_{F}}{d_{j}} \overleftarrow{\mathbf{H}}_{l j} \overleftarrow{\mathbf{V}}_{j}(n-1)\left(\overleftarrow{\mathbf{V}}_{j}(n-1)\right)^{*} \overleftarrow{\mathbf{H}}_{l j}^{*}
$$

5) Transmitters optimization phase: In the reverse training phase, the total interference power received at $S_{l}$ is

$$
\overleftarrow{I F}_{l}(n)=\operatorname{Tr}\left[\left(\overleftarrow{\mathbf{U}}_{l}(n)\right) * \overleftarrow{\mathbf{Q}}_{l}(n-1) \overleftarrow{\mathbf{U}}_{l}(n)\right]
$$

where $\overleftarrow{\mathbf{U}}_{l}(n)$ is the receiver filtering matrix of $\mathrm{S}_{l}$. Similarly, $\overleftarrow{\mathbf{u}}_{l}^{d}(n)\left(\forall d \in\left\{1, \ldots, d_{l}\right\}\right)$ is chosen as follows:

$$
\overleftarrow{\mathbf{u}}_{l}^{d}(n)=\nu^{d}\left[\overleftarrow{\mathbf{Q}}_{l}(n-1)\right]
$$

Next, $\mathrm{S}_{l}$ set $\mathbf{V}_{l}(n)=\overleftarrow{\mathbf{U}}_{l}(n)$ as its updated beamforming matrix in this training slot. Due to the channel reciprocity, such a choice would minimize the interference to unintended destinations in the forward direction [3].

An illustrative summary of the above five phases is shown in Fig. 2. The whole process would be repeated in each training slot, until the whole training is complete (i.e. after $N$ training slots). In the following section, we will explain the principles of the distributed power control strategy in detail.

\section{B. Distributed Power Control}

In the power updating phase of the $n$th training slot, the updated beamforming and filtering matrices at $\mathrm{S}_{k}$ and $\mathrm{D}_{k}$ are $\mathbf{V}_{k}(n-1)$ and $\mathbf{U}_{k}(n)$, respectively. For the simplicity of presentation, let $\mathbf{U}_{k}, \mathbf{V}_{k}$, and $p_{k}^{l}$ denote $\mathbf{U}_{k}(n), \mathbf{V}_{k}(n-1)$, and $p_{k}^{l}(n)\left(k \in\{1,2, \ldots, K\}, l \in\left\{1,2, \ldots, d_{k}\right\}\right)$, respectively. The SINR of the $l$ th stream at $\mathrm{D}_{k}$ is

$$
\operatorname{SINR}_{k}^{l}=\frac{\left|\left(\mathbf{u}_{k}^{l}\right)^{*} \mathbf{H}_{k k} \mathbf{v}_{k}^{l}\right|^{2} p_{k}^{l}}{\varphi_{k}^{l}(\mathbf{p})+N_{0}}
$$

where

$\varphi_{k}^{l}(\mathbf{p})=\sum_{j=1}^{K} \sum_{d=1}^{d_{j}}\left|\left(\mathbf{u}_{k}^{l}\right)^{*} \mathbf{H}_{k j} \mathbf{v}_{j}^{d}\right|^{2} p_{j}^{d}-\left|\left(\mathbf{u}_{k}^{l}\right)^{*} \mathbf{H}_{k k} \mathbf{v}_{k}^{l}\right|^{2} p_{k}^{l}$,

and $\mathbf{p}=\left[p_{1}^{1}, \ldots, p_{1}^{d_{1}}, \ldots, p_{K}^{1}, \ldots, p_{K}^{d_{K}}\right]^{T}$ is a $\left(\sum_{k=1}^{K} d_{k}\right) \times 1$ power vector. The mutual information of the source-destination pair $\mathrm{S}_{k}-\mathrm{D}_{k}$ is $\sum_{l=1}^{d_{k}} \log _{2}\left(1+\operatorname{SINR}_{k}^{l}\right)$. To guarantee successful transmission, the following conditions should be satisfied:

$$
\sum_{l=1}^{d_{k}} \log _{2}\left(1+\operatorname{SINR}_{k}^{l}\right) \geq R_{k} .
$$

The following requirement for each $l \in\left\{1, \ldots, d_{k}\right\}$ will guarantee the above condition to be met:

$$
\log _{2}\left(1+\operatorname{SINR}_{k}^{l}\right) \geq \frac{R_{k}}{d_{k}} .
$$

According to (12), (15) can be rewritten as a power constraint in the following format:

$$
p_{k}^{l} \geq I_{k}^{l}(\mathbf{p}),
$$

where

$$
I_{k}^{l}(\mathbf{p})=\frac{\left(2^{R_{k} / d_{k}}-1\right)\left(\varphi_{k}^{l}(\mathbf{p})+N_{0}\right)}{\left|\left(\mathbf{u}_{k}^{l}\right)^{*} \mathbf{H}_{k k} \mathbf{v}_{k}^{l}\right|^{2}} .
$$

Therefore, all power constraints can be represented as

$$
\mathbf{p} \succeq \mathbf{I}(\mathbf{p}),
$$




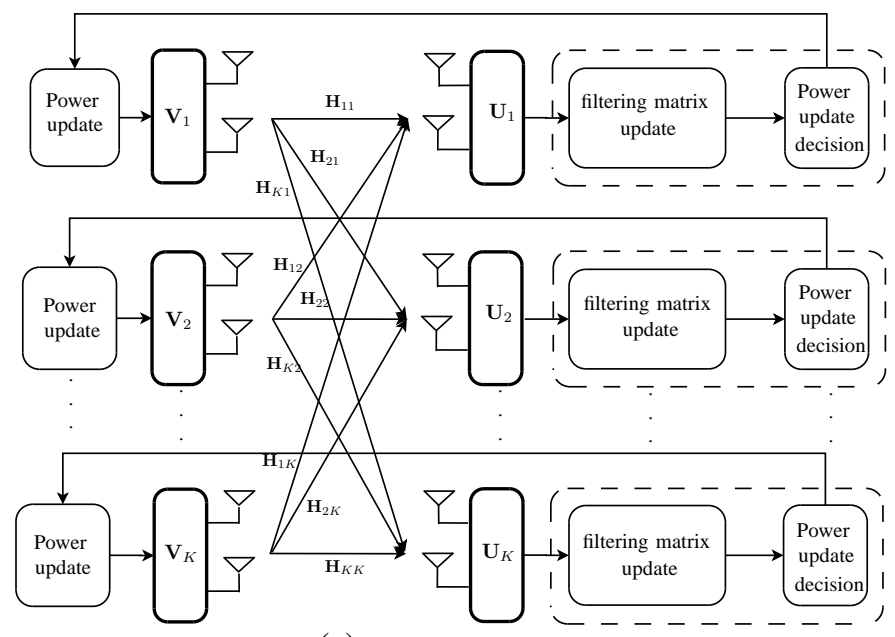

(a)

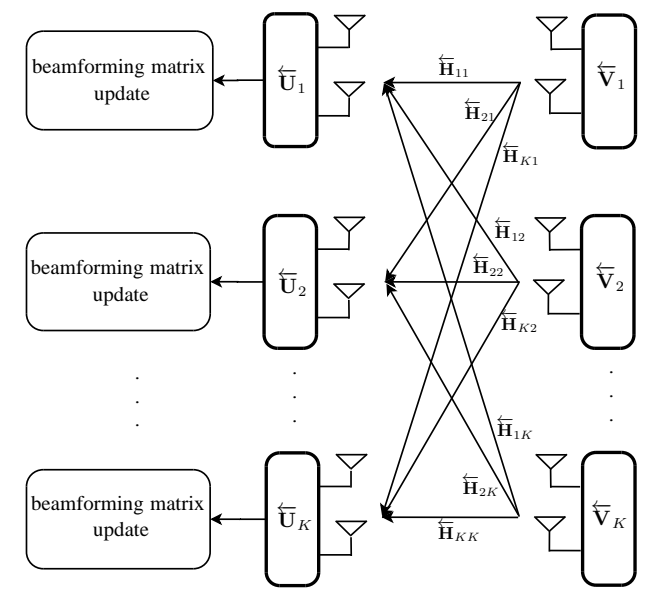

(b)

Fig. 2: (a) Updating the receiver filtering matrices and the powers (b) Updating the transmitter beamforming matrices

where $\mathbf{I}(\mathbf{p})=\left[I_{1}^{1}(\mathbf{p}), \ldots, I_{1}^{d_{1}}(\mathbf{p}), \ldots, I_{K}^{1}(\mathbf{p}), \ldots, I_{K}^{d_{K}}(\mathbf{p})\right]^{T}$ is called interference function, and the operator $\succeq$ denotes an element-wise inequality.

Definition 1: The set of positive power vectors which satisfy (15) in each training slot is defined as follows:

$$
\mathcal{P}_{\mathbf{H}}^{\mathrm{IA}}=\{\mathbf{p} \mid \mathbf{p} \succ \mathbf{0}, \mathbf{p} \succeq \mathbf{I}(\mathbf{p})\},
$$

where $\mathbf{I}(\mathbf{p})$ is the interference function defined in (18).

For a given set of transmitter beamforming and receiver filtering matrices, the power control problem can be formulated as the following optimization problem:

$$
\min _{\text {s.t. }} \sum_{\mathbf{p} \in \mathcal{P}_{\mathbf{H}}^{\text {II }}} \sum_{k=1}^{K} p_{k} .
$$

In the next part we present an iterative algorithm to find the minimum powers among all the feasible solutions.

1) Iterative power control: Recall that the transmitter beamforming matrices and the receiver filtering matrices are $\mathbf{V}_{k}$ and $\mathbf{U}_{k}(k \in\{1, \ldots, K\})$, respectively. We also use $p_{k}^{l}[i]$ $\left(l \in\left\{1, \ldots, d_{k}\right\}\right)$ to denote the calculated power at the $i$ th iteration. Before the first iteration starts, $\mathrm{S}_{k}$ sets its initial power values $p_{k}^{l}[0]=p_{k}^{l}(n-1)$. At the $(i-1)$ th $(i=1,2, \ldots)$ iteration, $\mathrm{D}_{k}$ computes $I_{k}^{l}(\mathbf{p}[i-1])$ using (17). Next, it sends these values back to $S_{k}$. This source will update its power values at the $i$ th iteration as follows:

$$
p_{k}^{l}[i]=I_{k}^{l}(\mathbf{p}[i-1]) .
$$

This iterative process continues until $i=M . \mathrm{S}_{k}$ updates the power of the $l$ th data stream at $n$th training slot as $p_{k}^{l}(n)=$ $p_{k}^{l}[M]$.

2) Convergence: The iterative power control algorithm in (21) for any initial vector $\mathbf{p}[0]$, generates a sequence of vectors $\mathbf{p}[1], \mathbf{p}[2], \ldots$. In this section, we answer two main questions regarding the convergence of this sequence. The first question is whether this sequence converges to a fixedpoint $\mathbf{p}^{*}\left(\mathbf{p}^{*}=\mathbf{I}\left(\mathbf{p}^{*}\right)\right)$. If it converges to a fixed-point, the second question is whether this fixed-point reflects the minimum required transmission powers.
Definition 2: $\mathbf{I}(\mathbf{p})$ is called a standard interference function if for all $\mathbf{p}, \mathbf{p}^{\prime} \succeq 0$, it satisfies the following conditions [7]:
1) Positivity : $\mathbf{I}(\mathbf{p}) \succ \mathbf{0}$;
2) Monotonicity : $\mathbf{I}(\mathbf{p}) \succeq \mathbf{I}\left(\mathbf{p}^{\prime}\right), \forall \mathbf{p} \succeq \mathbf{p}^{\prime}$;
3) Scalability: $\alpha \mathbf{I}(\mathbf{p}) \succ \mathbf{I}(\alpha \mathbf{p}), \forall \alpha>1$.

Theorem 1: If the problem in (20) is feasible $\left(\mathcal{P}_{\mathbf{H}}^{\mathrm{IA}} \neq \emptyset\right)$, for any initial power vector $\mathbf{p}[0]$, the recursive equation in (21) generates a sequence of vectors which converges to a unique fixed-point $\mathbf{p}^{*}$. The fixed-point corresponds to the solution of the problem in (20).

Proof: First, we prove that the function $\mathbf{I}(\mathbf{p})$ introduced in (18) is a standard interference function, by showing that it satisfies the conditions given in Definition 2. To simplify this verification we rewrite $I_{k}^{l}(\mathbf{p})$ as $I_{k}^{l}(\mathbf{p})=L \cdot\left(\varphi_{k}^{l}(\mathbf{p})+N_{0}\right)$, where $L=\frac{2^{R_{k} / d_{k}-1}}{\left|\left(\mathbf{u}_{k}^{l}\right) * \mathbf{H}_{k k} \mathbf{v}_{k}^{l}\right|^{2}}>0$.

1) To check the positivity condition we have:

$$
I_{k}^{l}(\mathbf{p})=L \cdot\left(\varphi_{k}^{l}(\mathbf{p})+N_{0}\right) \stackrel{(a)}{\geq} L N_{0}>0,
$$

where (a) follows the fact that $\varphi_{k}^{l}(\mathbf{p}) \geq 0$. Therefore, the positivity condition is satisfied.

2) To verify the monotonicity condition, we assume $\mathbf{p} \succeq \mathbf{p}^{\prime}$ :

$$
\begin{aligned}
\mathbf{p} \succeq \mathbf{p}^{\prime} & \stackrel{(a)}{\Rightarrow} p_{j}^{l}\left|\left(\mathbf{u}_{k}^{l}\right)^{*} \mathbf{H}_{k j} \mathbf{v}_{j}^{l}\right|^{2} \geq{p^{\prime}}_{j}\left|\left(\mathbf{u}_{k}^{l}\right)^{*} \mathbf{H}_{k j} \mathbf{v}_{j}^{l}\right|^{2}, \forall l, k, j \\
& \stackrel{(b)}{\Rightarrow} \varphi_{k}^{l}(\mathbf{p}) \geq \varphi_{k}^{l}\left(\mathbf{p}^{\prime}\right), \quad \forall l, k \\
& \stackrel{(c)}{\Rightarrow} I_{k}(\mathbf{p}) \geq I_{k}\left(\mathbf{p}^{\prime}\right), \forall k \Rightarrow \mathbf{I}(\mathbf{p}) \succeq \mathbf{I}\left(\mathbf{p}^{\prime}\right)
\end{aligned}
$$

where $(a)$ follows the fact that $\left|\left(\mathbf{u}_{k}^{l}\right)^{*} \mathbf{H}_{k j} \mathbf{v}_{j}^{l}\right|^{2} \geq 0 ;(b)$ follows the definition of $\varphi_{k}^{l}(\mathbf{p})$ in (13); (c) follows the definition in (17) and the fact that $\left|\left(\mathbf{u}_{k}^{l}\right)^{*} \mathbf{H}_{k k} \mathbf{v}_{k}^{l}\right|^{2}>0$. Therefore, the monotonicity condition is satisfied.

3 ) To check the scalability condition we assume $\alpha>1$, thus:

$$
\begin{aligned}
I_{k}(\alpha \mathbf{p}) & =L \cdot\left(\varphi_{k}^{l}(\alpha \mathbf{p})+N_{0}\right) \\
& =L \cdot\left(\alpha \varphi_{k}^{l}(\mathbf{p})+N_{0}\right) \\
& <\alpha L \cdot\left(\varphi_{k}^{l}(\mathbf{p})+N_{0}\right)=\alpha \mathbf{I}_{k}(\mathbf{p}) .
\end{aligned}
$$




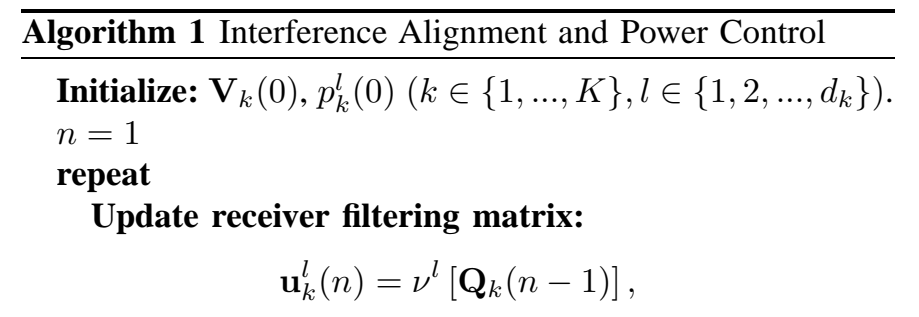

where

$$
\mathbf{Q}_{k}(n-1)=\sum_{\substack{j=1 \\ j \neq k}}^{K} \sum_{\substack{d_{k} \\ p_{j}}}^{d}(n-1) \mathbf{H}_{k j} \mathbf{v}_{j}^{d}(n-1)\left(\mathbf{v}_{j}^{d}(n-1)\right)^{*} \mathbf{H}_{k j}^{*} .
$$

Update transmission power:

$$
p_{k}^{l}(n)=\frac{\left(2^{R_{k} / d_{k}}-1\right)\left(\varphi_{k}^{l}(n-1)+N_{0}\right)}{\left|\left(\mathbf{u}_{k}^{l}(n)\right)^{*} \mathbf{H}_{k k} \mathbf{v}_{k}^{l}(n-1)\right|^{2}},
$$

where

$$
\begin{aligned}
\varphi_{k}^{l}(n-1) & =\sum_{j=1}^{K} \sum_{d=1}^{d_{j}}\left|\left(\mathbf{u}_{k}^{d}(n)\right)^{*} \mathbf{H}_{k j} \mathbf{v}_{j}^{d}(n-1)\right|^{2} p_{j}^{d}(n-1) \\
& -\left|\left(\mathbf{u}_{k}^{l}(n)\right)^{*} \mathbf{H}_{k k} \mathbf{v}_{k}^{l}(n-1)\right|^{2} p_{k}^{l}(n-1) .
\end{aligned}
$$

Set reverse beamforming matrix: $\overleftarrow{\mathbf{V}}_{k}(n)=\mathbf{U}_{k}(n)$.

Update transmitter beamforming matrix:

$$
\overleftarrow{\mathbf{u}}_{k}^{l}(n)=\nu^{l}\left[\overleftarrow{\mathbf{Q}}_{k}(n)\right]
$$

where $\overleftarrow{\mathbf{Q}}_{k}(n)=\sum_{j=1, j \neq k}^{K} \frac{p_{F}}{d_{j}} \overleftarrow{\mathbf{H}}_{k j} \overleftarrow{\mathbf{V}}_{j}(n)\left(\overleftarrow{\mathbf{V}}_{j}(n)\right)^{*} \overleftarrow{\mathbf{H}}_{k j}^{*}$

Set beamforming matrix: $\mathbf{V}_{k}(n)=\overleftarrow{\mathbf{U}}_{k}(n)$.

Increase the training slot index $n=n+1$.

until $n=N$

This condition is satisfied for any $k \in\{1,2, \ldots, K\}$ and the scalability condition is satisfied.

Thus, we can conclude that $\mathbf{I}(\mathbf{p})$ given in (18) is a standard interference function. According to Theorem 2 in [7], if the problem is feasible $\left(\mathcal{P}_{\mathbf{H}}^{\mathrm{IA}} \neq \emptyset\right)$, for any initial power vector $\mathbf{p}[0]$ the algorithm in (21) converges to a unique fixed-point $\mathbf{p}^{*}$. Lemma 1 in [7] implies that this fixed-point is the solution with the minimum required transmission power for each source. The feasibility of this problem has been investigated in [12], and it has been shown how it depends on the rates, beamforming and the filtering matrices.

3) Joint interference alignment and power control: The above power control scheme requires iterative calculations to be performed during the power updating phase of each training slot. This may increase the complexity of the transmission scheme. Nevertheless, this scheme can be simplified such that during the power updating phase, each source updates its transmission powers only once. Two different algorithms are proposed to do this. The difference of them lies in different assumptions regarding the available feedback channels to send the calculated power values from each destination to the cor-
Algorithm 2 Interference Alignment and Power Control: onebit feedback signal for each power value

Initialize: $\mathbf{V}_{k}(0), p_{k}^{l}(0)\left(k \in\{1, \ldots, K\}, l \in\left\{1,2, \ldots, d_{k}\right\}\right)$.

Set $0<\alpha<1, \Delta>1, n=1$.

repeat

Update receiver filtering matrix:

$$
\mathbf{u}_{k}^{l}(n)=\nu^{l}\left[\mathbf{Q}_{k}(n-1)\right],
$$

where

$$
\mathbf{Q}_{k}(n-1)=\sum_{\substack{j=1 d=1 \\ j \neq k}}^{K} \sum_{j}^{d_{k}} p_{j}^{d}(n-1) \mathbf{H}_{k j} \mathbf{v}_{j}^{d}(n-1)\left(\mathbf{v}_{j}^{d}(n-1)\right)^{*} \mathbf{H}_{k j}^{*} .
$$

Update transmission power:

$$
\begin{aligned}
& \text { if } p_{k}^{l}(n-1)<\frac{\left(2^{R_{k}} / d_{k}-1\right)\left(\varphi_{k}^{l}(n-1)+N_{0}\right)}{\left|\left(\mathbf{u}_{k}^{l}(n)\right)^{*} \mathbf{H}_{k k} \mathbf{v}_{k}^{l}(n-1)\right|^{2}} \text {, then } \\
& \quad p_{k}^{l}(n)=(1-\alpha) p_{k}^{l}(n-1)+\alpha \Delta p_{k}^{l}(n-1) \\
& \text { else } \\
& \quad p_{k}^{l}(n)=(1-\alpha) p_{k}^{l}(n-1)+\frac{\alpha}{\Delta} p_{k}^{l}(n-1)
\end{aligned}
$$

end if

where

$$
\begin{aligned}
\varphi_{k}^{l}(n-1) & =\sum_{j=1}^{K} \sum_{d=1}^{d_{j}}\left|\left(\mathbf{u}_{k}^{d}(n)\right)^{*} \mathbf{H}_{k j} \mathbf{v}_{j}^{d}(n-1)\right|^{2} p_{j}^{d}(n-1) \\
& -\left|\left(\mathbf{u}_{k}^{l}(n)\right)^{*} \mathbf{H}_{k k} \mathbf{v}_{k}^{l}(n-1)\right|^{2} p_{k}^{l}(n-1) .
\end{aligned}
$$

Set the reverse beamforming matrix $\overleftarrow{\mathrm{V}}_{k}(n)=\mathbf{U}_{k}(n)$. Update transmitter beamforming matrix:

$$
\overleftarrow{\mathbf{u}}_{k}^{l}(n)=\nu^{l}\left[\overleftarrow{\mathbf{Q}}_{k}(n)\right]
$$

where $\overleftarrow{\mathbf{Q}}_{k}(n)=\sum_{j=1, j \neq k}^{K} \frac{p_{F}}{d_{j}} \overleftarrow{\mathbf{H}}_{k j} \overleftarrow{\mathbf{V}}_{j}(n)\left(\overleftarrow{\mathbf{V}}_{j}(n)\right)^{*} \overleftarrow{\mathbf{H}}_{k j}^{*}$

Set the beamforming matrix: $\mathbf{V}_{k}(n)=\overleftarrow{\mathbf{U}}_{k}(n)$.

Increase the iteration index $n=n+1$.

until $n=N$

responding source. In Algorithm 1, during a training slot each destination updates its receiver filtering matrix and computes the required transmission powers of the corresponding source. Next, it feeds back these values to the corresponding source. Each source updates its transmission powers according to the received feedback signal. This algorithm requires a perfect feedback channel from each destination to the corresponding source. A simpler solution is proposed in Algorithm 2, which only requires a one-bit feedback signal, corresponding to each data stream, from a destination to the corresponding source. Thus, each destination informs the corresponding source to either increase or decrease the transmission powers. The updated power is a summation of the power in the previous training slot and its scaled version which are weighted by $\alpha$ $(0<\alpha<1)$ and $(1-\alpha)$, respectively. Depending on the value of feedback signal, the scaling factor is either $\Delta(\Delta>1)$ or $1 / \Delta . \alpha$ and $\Delta$ are design parameters which control the stability and convergence speed of the algorithm. 


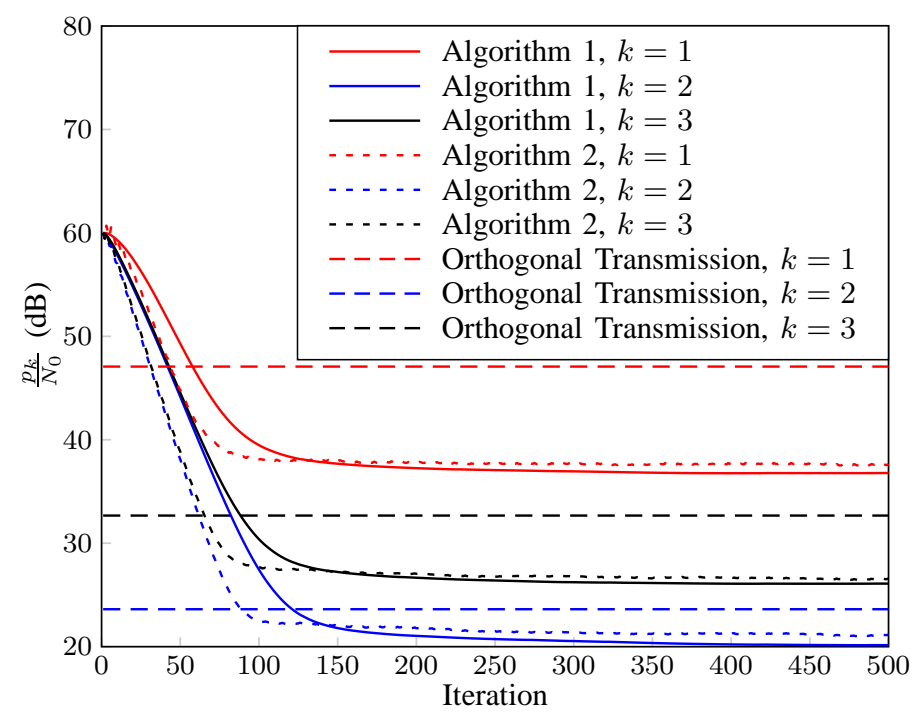

Fig. 3: Power of source $S_{k}$ versus number of iterations

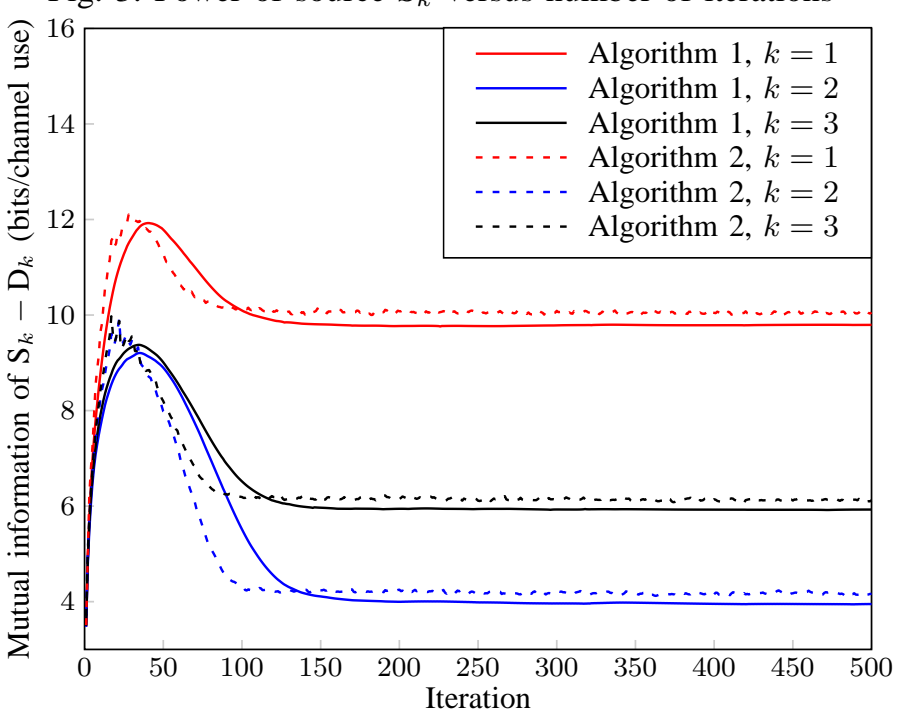

Fig. 4: Mutual information of source-destination pair $S_{k}-D_{k}$ versus number of iterations.

\section{Performance Evaluation}

In this section, we numerically evaluate the performance of Algorithm 1 and Algorithm 2. For comparison, we also provide results for the orthogonal transmission scheme, in which the sources are orthogonally activated to directly avoid interference. Consequently, the transmitter beamforming and the receiver filtering matrix design for each source-destination pair is simplified to that for point-to-point MIMO systems. Thus, the solution of the power control for each interferencefree channel simplifies to water-filling.

As an example, we consider a three-user network where each terminal is equipped with two antennas. When the orthogonal transmission is performed, only one source is active at a time and transmits two data streams, while the others are silent. In our proposed algorithms, all the sources are concurrently activated while each of them transmits one data stream $\left(d_{k}=1, \forall k \in\{1,2, \ldots, K\}\right)$. The rates are fixed as
$\mathbf{R}=\left[\begin{array}{lll}10 & 4 & 6\end{array}\right]$ (bits/channel use), and $\frac{p_{k}^{l}(0)}{N_{0}}=60(\mathrm{~dB})$. The number of different channel realizations is set to $10^{5}$.

Fig. 3 shows the average required powers in each iteration. We can see that Algorithm 1 and Algorithm 2 have a similar convergence behavior. By increasing the number of iterations, the powers converge. Compared with the orthogonal transmission, our algorithms requires substantially lower powers.

Fig. 4 displays the mutual information of the three sourcedestination pairs, which converge to the corresponding desired transmission rates as the number of iterations increases. Both figures illustrate how the proposed algorithms reduce transmission powers to the minimum required levels while successful transmissions at given rates are still possible.

\section{CONClusion}

In this paper we have addressed transceiver design and power control for time-invariant MIMO interference networks to guarantee successful communication at given rates. We have proposed two distributed iterative algorithms which require only local CSI at each terminal. The transmitter beamforming and the receiver filtering matrices are designed to realize interference alignment. In addition, power control is performed to assign the minimum required powers. Performance evaluations confirm that these algorithms require substantially lower powers compared to the conventional orthogonal transmission.

\section{REFERENCES}

[1] M. A. Maddah-Ali, A. S. Motahari, and A. K. Khandani, "Communication over MIMO X channels: Interference alignment, decomposition, and performance analysis," IEEE Trans. Inf. Theory, vol. 54, no. 8, pp. 3457-3470, Aug. 2008

[2] V. R. Cadambe and S. A. Jafar, "Interference alignment and degrees of freedom of the K-user interference channel," IEEE Trans. Inf. Theory, vol. 54, no. 8, pp. 3425 -3441, Aug. 2008.

[3] K. Gomadam, V. R. Cadambe, and S. A. Jafar, "A distributed numerical approach to interference alignment and applications to wireless interference networks," IEEE Trans. Inf. Theory, vol. 57, no. 6, pp. $3309-$ 3322, Jun. 2011.

[4] X. Wang, G. B. Giannakis, and A. G. Marques, "A unified approach to QoS-guaranteed scheduling for channel-adaptive wireless networks," Proc. IEEE, vol. 95, no. 12, pp. 2410-2431, Dec. 2007.

[5] J. Zander, "Distributed cochannel interference control in cellular radio systems interference," IEEE Trans. Veh. Technol., vol. 41, no. 3, pp. 305-311, Aug. 1992.

[6] G. J. Foschini and Z. Miljanic, "A simple distributed autonomous power control algorithm and its convergence," IEEE Trans. Veh. Technol., vol. 42, no. 4, pp. 641-646, Nov. 1993.

[7] R. D. Yates, "A framework for uplink power control in cellular radio systems," IEEE J. Sel. Areas Commun., vol. 13, no. 7, pp. 1341-1347, Sep. 1995.

[8] M. Schubert and H. Boche, "Solution of the multiuser downlink beamforming problem with individual SINR constraints," IEEE Trans. Veh. Technol., vol. 53, no. 1, pp. 18-28, Jan. 2004.

[9] F. Rashid-Farrokhi, K. J. R. Liu, and L. Tassiulas, "Transmit beamforming and power control for cellular wireless systems," IEEE J. Sel. Areas Commun., vol. 16, no. 8, p. 14371450, Oct. 1998.

[10] C. W. Tan, M. Chiang, and R. Srikant, "Maximizing sum rate and minimizing MSE on multiuser downlink: Optimality, fast algorithms, and equivalence via max-min SINR," IEEE Trans. Signal Process., vol. 59, no. 12, pp. 6127-6143, Dec. 2011.

[11] C. M. Yetis, T. Gou, S. A. Jafar, and A. H. Kayran, "Feasibility conditions for interference alignment," in IEEE Global Telecommunications Conference (GLOBECOM'09), Hawaii, USA, Nov. 2009.

[12] H. Farhadi, "Interference alignment and power control for wireless interference networks," Licentiate thesis, KTH Royal Institute of Technology, Sep. 2012. 